\title{
No evidence of Borrelia mayonii in an endemic area for Lyme borreliosis in France
}

\author{
Pierre H. Boyer', Sylvie J. De Martino ${ }^{1,2}$, Yves Hansmann ${ }^{3}$, Laurence Zilliox ${ }^{2}$, Nathalie Boulanger ${ }^{1,2}$ \\ and Benoit Jaulhac ${ }^{1,2^{*}}$ (iD
}

\begin{abstract}
Background: Borrelia mayonii is currently the latest species belonging to the Borrelia burgdorferi (sensu lato) complex to be discovered. Interestingly it is involved in human pathology causing a high fever. We looked for its presence in post- tick bite febrile patients as well as in Ixodes ricinus ticks in an endemic area of France.

Results: After ensuring that our molecular technics correctly detected B. mayonii, 575 patients and 3,122 Ixodes ricinus nymphs were tested. Neither B. mayonii nor another species of the B. burgdorferi (s.l.) complex previously not reported in Europe has been identified.
\end{abstract}

Conclusions: For now, B. mayonii seems to be an epiphenomenon. However, its discovery broadens the etiology of post-Ixodes bite febrile syndromes.

Keywords: Lyme borreliosis, Borrelia mayonii, Borrelia burgdorferi (sensu lato), Fever

\section{Background}

Lyme borreliosis is reported as the most common tickborne disease in the United States [1] and all the northern hemisphere. Since the early research of Baranton and colleagues [2], several species belonging to the Borrelia burgdorferi (sensu lato) (s.l.) complex have been described. Four are currently the main etiological agents of human Lyme borreliosis in Europe [1], and Borrelia burgdorferi (sensu stricto) (s.s.) was considered for a long time as the only species isolated from human clinical samples on the American continent [3, 4]. The recent discovery of Borrelia mayonii [4] questions this idea. This newly identified bacterium seems to display unusual clinical characteristics. Unlike the other species of the B. burgdorferi (s.l.) complex, uncommonly high fever due to unusual high spirochetemia as well as cutaneous rashes was observed in patients infected by $B$. mayonii [4]. It is, therefore, the first time that a member of the B. burgdorferi (s.l.) complex had been shown to induce high fever as reported for relapsing fever

\footnotetext{
* Correspondence: jaulhac@unistra.fr

${ }^{1}$ Early Bacterial Virulence: Lyme borreliosis Group, Université de Strasbourg, CHRU Strasbourg, Fédération de Médecine Translationnelle de Strasbourg, VBP EA 7290, F-67000 Strasbourg, France

${ }^{2}$ French National Reference Center for Borrelia, Hôpitaux Universitaires de Strasbourg, Strasbourg, France

Full list of author information is available at the end of the article
}

Borrelia, thus expanding the field of symptoms found in Lyme borreliosis. We, therefore, investigated its potential presence in patients presenting with fever after a suspicion of tick bite in Alsace, a French region endemic for Lyme borreliosis [5], and also in Ixodes ricinus, the vector of $B$. burgdorferi (s.l.) in western Europe [1].

\section{Methods}

DNA extracts from whole blood samples of 575 febrile patients sent to our laboratory between January 2010 and July 2016 were studied. All patients included in this study hailed from northeastern France and developed either a fever above $38{ }^{\circ} \mathrm{C}$ within three weeks after a tick bite or fever of unknown origin with exposure to a tick bite in an endemic area of Lyme borreliosis during spring and summer (April to October). All the subjects enrolled in this study completed a written informed consent. In parallel, 3,122 I. ricinus questing nymphs collected monthly by flagging in four separate locations in Alsace from April 2013 to December 2015, were investigated.

Both patients and ticks were tested for B. burgdorferi (s.l.) using an in-house Taqman ${ }^{\circ}$ real-time PCR assay [6], targeting a conserved region of the flagellin gene $(f l a)$ of the B. burgdorferi (s.l.) complex. Borrelia turdii whole DNA at a concentration of $1 \mathrm{pg} / \mu \mathrm{l}$ was used as a positive 
control. The genotyping of B. burgdorferi (s.l.) species was performed on positive samples with another realtime PCR assay using hybridization probes targeting species-specific regions of the fla gene [6]. To ascertain that the techniques correctly detect $B$. mayonii, we firstly aligned our primers and probes in silico with the $476 \mathrm{bp}$ fragment of the corresponding fla gene (GenBank KR154295.1). Then, the B. mayonii type strain ATCC BAA 2743 was cultured in the BSK-H medium (Sigma-Aldrich, Saint Quentin Fallavier, France). Borrelia mayonii whole DNA was extracted with the MagNA Pure system (Roche Diagnostics, Meylan, France). After extraction, DNA concentration was measured by NanoDrop 1000 Fluorospectrometer (Thermo Fischer Scientific, Villebon sur Yvette, France). To evaluate the sensitivity of our technique, a dilution range was tested.

\section{Results}

Analyses of results showed that $B$. mayonii was correctly detected by our B. burgdorferi (s.l.) PCR assay up to a concentration of 2 bacteria/ $\mu \mathrm{L}$; similar results were found with the main pathogenic species. Moreover, $B$. mayonii is never misidentified by our typing assay and amplicon sequencing would have confirmed $B$. mayonii identification. Only one out of the 575 blood samples tested from the post-tick bite febrile patients was positive for B. burgdorferi (s.l.). This sample was further identified as being $B$. afzelii by our typing assay and confirmed by sequencing. Interestingly, this patient had a co-infection with the tick-borne encephalitis virus (TBEv). He had, without previous vaccination, anti-TBEv IgG and IgM and thus met the ECDC Laboratory criteria for a confirmed TBE case [7]. On another serum tested one month later, the anti-TBEv IgM had disappeared. The involvement of $B$. afzelii in clinical manifestations of this patient is questionable since TBEv infection is known to induce fever and headache [8]. Among the 3,122 tested nymphs, $12.7 \%$ were infected with B. burgdorferi (s.l.), and only Borrelia species commonly found in this area were identified, such as B. afzelii, B. garinii and B. burgdorferi (s.s.). All ticks were negative for $B$. mayonii, and no other Borrelia species was identified.

\section{Discussion}

The discovery of $B$. mayonii questions the paradigm of the absence of fever in Lyme disease. Considered as unusual [1,9-11], high fever is not a cardinal symptom of Lyme disease, but three out of the six patients previously described by Pritt and colleagues [4] had fever above $38{ }^{\circ} \mathrm{C}$ with even two exceeding $39{ }^{\circ} \mathrm{C}$. This new clinical picture broadens the symptom spectrum of Lyme disease, justifying the extension of investigation to an additional member of the B. burgdorferi (s.l.) complex in febrile patients in France. Neither B. mayonii nor another new species of the B. burgdorferi (s.l.) complex has been identified in our patient cohort. For now, B. mayonii should be considered an epiphenomenon [4]. The vector competence of Ixodes scapularis has been clearly demonstrated [12], but the competence of I. ricinus and I. persulcatus for this new Borrelia species, remains to be proven. Nevertheless, potential new members of the B. burgdorferi (s.l.) complex could be responsible for high fever and should be included in post-tick bite febrile syndrome active surveillance. This new pathogen monitoring should be initiated on European, Asian and American continents along with other etiological agents of post-tick bite fever.

\section{Abbreviations \\ fla: Flagellin}

\section{Acknowledgements}

We warmly thank Marie-Christine Michellet, an ex-English teacher, for her help in the revision of this article.

\section{Funding}

Funding for this study was partly provided by the Programme Hospitalier de Recherche Clinique (PHRC) National No 3960 and partly by the French

National Reference Center for Borrelia.

\section{Availability of data and materials}

Data and materials can be obtained on request to the corresponding author.

\section{Author's contributions}

PB performed in silico analysis, experiments on humans, collected ticks, wrote the paper. LZ collected ticks and extracted DNA from ticks. SJDM and $\mathrm{YH}$ equally provided human samples and clinical data. NB, collected ticks and corrected the paper. BJ directly supervised PB for the experiments and corrected the paper and. All authors read and approved the final manuscript.

\section{Competing interests}

The authors declare that they have no competing interests.

\section{Consent for publication}

Not applicable.

\section{Ethics approval and consent to participate}

The present work was approved by the European Clinical Trials Database (No EUDRACT: 2009 A00869-48). All the subjects enrolled in this study completed a written informed consent.

\section{Publisher's Note}

Springer Nature remains neutral with regard to jurisdictional claims in published maps and institutional affiliations.

\section{Author details}

'Early Bacterial Virulence: Lyme borreliosis Group, Université de Strasbourg, CHRU Strasbourg, Fédération de Médecine Translationnelle de Strasbourg, VBP EA 7290, F-67000 Strasbourg, France. ${ }^{2}$ French National Reference Center for Borrelia, Hôpitaux Universitaires de Strasbourg, Strasbourg, France. ${ }^{3}$ Department of Infectious and Tropical Diseases, Hôpitaux Universitaires de Strasbourg, Strasbourg, France.

Received: 24 January 2017 Accepted: 22 May 2017

Published online: 05 June 2017

\section{References}

1. Stanek G, Wormser GP, Gray J, Strle F. Lyme borreliosis. Lancet Lond Engl. 2012;379:461-73.

2. Baranton G, Postic D, Saint Girons I, Boerlin P, Piffaretti J-C, Assous M, et al Delineation of Borrelia burgdorferi sensu stricto, Borrelia garinii sp. nov., and 
group VS461 associated with Lyme borreliosis. Int J Syst Evol Microbiol. 1992:42:378-83.

3. Rudenko N, Golovchenko M, Vancova M, Clark K, Grubhoffer L, Oliver Jr JH. Isolation of live Borrelia burgdorferi sensu lato spirochaetes from patients with undefined disorders and symptoms not typical for Lyme borreliosis. Clin Microbiol Infect. 2016;22:267.e9-267.e15.

4. Pritt BS, Mead PS, Johnson DKH, Neitzel DF, Respicio-Kingry LB, Davis JP, et al Identification of a novel pathogenic Borrelia species causing Lyme borreliosis with unusually high spirochaetaemia: a descriptive study. Lancet Infect Dis. 2016;16:556-64.

5. Vandenesch A, Turbelin C, Couturier E, Arena C, Jaulhac B, Ferquel E, et al. Incidence and hospitalisation rates of Lyme borreliosis, France, 2004 to 2012. Euro Surveill. 2014;19(34).

6. Hidri N, Barraud O, de Martino S, Garnier F, Paraf F, Martin C, et al. Lyme endocarditis. Clin Microbiol Infect. 2012:18:531-2.

7. Second expert consultation on tick-borne diseases with emphasis on Lyme borreliosis and tick-borne encephalitis [Internet]. European Centre for Disease Prevention and Control; 2012. Available from: http://ecdc.europa.eu/ en/publications/Publications/Tick-borne-diseases-meeting-report.pdf. Accessed 3 Mar 2017.

8. Lindquist L, Vapalahti O. Tick-borne encephalitis. Lancet. 2008;371:1861-71.

9. Strle F, Ruzić-Sabljić E, Cimperman J, Lotric-Furlan S, Maraspin V. Comparison of findings for patients with Borrelia garinii and Borrelia afzelii isolated from cerebrospinal fluid. Clin Infect Dis. 2006;43:704-10.

10. Strle F, Ružić-Sabljić E, Logar M, Maraspin V, Lotrič-Furlan S, Cimperman J, et al. Comparison of erythema migrans caused by Borrelia burgdorferi and Borrelia garinii. Vector Borne Zoonotic Dis Larchmt N. 2011;11:1253-8.

11. Logar M, Ruzić-Sabljić E, Maraspin V, Lotric-Furlan S, Cimperman J, Jurca T, et al. Comparison of erythema migrans caused by Borrelia afzelii and Borrelia garinii. Infection. 2004;32:15-9.

12. Dolan MC, Hojgaard A, Hoxmeier JC, Replogle A, Respicio-Kingry LB, Sexton C, et al. Vector competence of the blacklegged tick, Ixodes scapularis, for the recently recognized Lyme borreliosis spirochete Candidatus Borrelia mayonii. Ticks Tick-Borne Dis. 2016;5:665-9.

\section{Submit your next manuscript to BioMed Central and we will help you at every step:}

- We accept pre-submission inquiries

- Our selector tool helps you to find the most relevant journal

- We provide round the clock customer support

- Convenient online submission

- Thorough peer review

- Inclusion in PubMed and all major indexing services

- Maximum visibility for your research

Submit your manuscript at www.biomedcentral.com/submit

) Biomed Central 